\title{
The effect of pre-seasonal strength training on central hemodynamics and cardiac function in elite powerlifting athletes
}

This article was published in the following Dove Press journal:

Research Reports in Clinical Cardiology

\author{
Janno Jürgenson ${ }^{1,2}$ \\ Martin Serg ${ }^{3,4}$ \\ Priit Kampus ${ }^{3,4}$ \\ Jaak Kals ${ }^{4-6}$ \\ Piibe Muda ${ }^{7}$ \\ Maksim Zagura ${ }^{3,4}$ \\ Mehis Viru' \\ Mihkel Zilmer ${ }^{4,6}$ \\ Jaan $\mathrm{Eha}^{3,4,7}$ \\ Eve Unt ${ }^{2,3,8,9}$ \\ 'Institute of Sport Sciences and \\ Physiotherapy, Medical Faculty, University \\ of Tartu, Tartu, Estonia; ${ }^{2}$ Estonian Centre \\ of Behavioural and Health Sciences, \\ University of Tartu, Tartu, Estonia; \\ ${ }^{3}$ Department of Cardiology, Institute of \\ Clinical Medicine, Medical Faculty, \\ University of Tartu, Tartu, Estonia; \\ ${ }^{4}$ Endothelial Centre, University of Tartu, \\ Tartu, Estonia; ${ }^{5}$ Department of Surgery, \\ Institute of Clinical Medicine, Medical \\ Faculty, University of Tartu, Tartu, \\ Estonia; ${ }^{6}$ Department of Biochemistry, \\ Institute of Biomedicine and Translational \\ Medicine, Centre of Excellence for \\ Genomics and Translational Medicine, \\ Medical Faculty, University of Tartu, \\ Tartu, Estonia; ${ }^{7}$ Heart Clinic, Tartu \\ University Hospital, Tartu, Estonia; \\ ${ }^{8}$ Department of Sports Medicine and \\ Rehabilitation, Institute of Clinical \\ Medicine, Medical Faculty, University of \\ Tartu, Tartu, Estonia; ${ }^{9}$ Sports Medicine \\ and Rehabilitation Clinic, Tartu University \\ Hospital, Tartu, Estonia
}

Correspondence: Janno Jürgenson Institute of Sport Sciences and

Physiotherapy, Medical Faculty, University of Tartu, Str Ülikooli I8, Tartu 50090,

Estonia

Tel +37252062II

Email jannosport@gmail.com
Aim: The purpose of this study was to evaluate the effect of a pre-seasonal 12-week supervised strength training program (SSTP) on arterial stiffness, brachial and central blood pressure, and systolic and diastolic function of the heart in elite powerlifting athletes. Methods: A descriptive study was performed in 19 elite male powerlifters, who exercised for 12 weeks, four days per week with an intensity of $60-90 \%$ assessed from 1 repetition maximum and 90-120 min per session. Carotid-femoral pulse wave velocity (cfPWV), augmentation index and central blood pressure were measured by applanation tonometry using the SphygmoCor device, and an echocardiographic examination was performed at baseline and after 12-week SSTP.

Results: Subjects' mean brachial and central systolic blood pressure decreased significantly after the training period $(132.3 \pm 8.8$ vs $124.3 \pm 8.7 \mathrm{mmHg}, p=0.002$ and $110.1 \pm 7.7$ vs 104.5 $\pm 8.7 \mathrm{mmHg}, p=0.008$, respectively). Strength training significantly improved systolic tricuspid annular velocity and mitral E/e' ratio $(p<0.05)$. There were no significant differences in cfPWV and augmentation index at baseline and after SSTP.

Conclusion: The 12-week supervised strength pre-seasonal training program significantly decreased brachial and central systolic blood pressure in male elite powerlifting athletes. The strength training program decreased left ventricular filling pressure and improved right ventricular systolic function. No significant effect of strength training on aortic stiffness was found.

Keywords: pulse wave velocity, resistance training, cardiovascular health, echocardiography

\section{Introduction}

The blood pressure (BP) varies throughout the arterial tree due to pulse wave reflection and arterial stiffness. ${ }^{1}$ Elevated BP is a common abnormal finding during the periodic health evaluation of athletes. ${ }^{2}$ Strength-trained athletes and weightlifters have a higher prevalence of hypertension as compared to endurance athletes. ${ }^{3,4}$ Elevated BP leads to increased afterload on the heart causing left ventricular hypertrophy, which is an independent predictor of cardiovascular risk. ${ }^{4,5}$ Furthermore, there is an increasing amount of evidence that central BP is better related to target organ damage and cardiovascular events than brachial BP. ${ }^{6,7}$

It has been previously demonstrated that arterial stiffness is associated with a number of traditional and novel cardiovascular risk factors and target organ damage in patients with atherosclerosis and hypertension. ${ }^{8,9}$ There is also evidence that a reduction in arterial stiffness results in reduced incidence of cardiovascular events. $^{10}$ 
Data have shown the beneficial cardiovascular effect of aerobic exercise is explained by amelioration of traditional as well as novel risk factors. ${ }^{11}$ Aerobic exercise augments endothelium-dependent vasodilation, ${ }^{12}$ which in turn may reduce aortic stiffness. ${ }^{13}$ At present, little is known about the effects of high-intensity strength training on central blood pressure and arterial stiffness. High-intensity strength training involves slow-speed contractions of the muscles, which are related to transient mechanical compression of resistant vessels, increasing peripheral vascular resistance and left ventricular pressure overload during exercise. ${ }^{14}$ It is still under discussion whether chronic increase in afterload may lead to concentric ventricular hypertrophy. ${ }^{15}$ Recent meta-analysis ${ }^{16}$ revealed that acute resistance training has an adverse effect on arterial stiffness due to cardiovascular as well as non-cardiovascular mechanisms. In regard to systematic strength training, available studies have shown stiffer arteries in strengthtrained athletes, ${ }^{17,18}$ while other data have shown a beneficial effect of resistance training on aortic stiffness. ${ }^{19,20}$ Inconsistent results may depend on many factors. Many of these studies were performed in relatively small samples in different age and sex groups and by a large variation in training intensities. In addition, most of the intervention studies were performed in untrained, recreational level or pre-hypertensive individuals. Thus, available studies have not revealed a clear understanding of the impact of the combination of dynamic and isometric resistance training, also known as strength training, on central blood pressure and arterial stiffness in healthy individuals. ${ }^{17-27}$ Furthermore, the effect of elite-level strength training for lowering BP and target organ damage is still unclear.

Therefore, we aimed to investigate the effect of a preseasonal strength training program on brachial and central $\mathrm{BP}$, arterial stiffness as well as heart structure and function in elite powerlifting athletes.

\section{Material and methods Subjects}

A total of 20 voluntary male elite powerlifting athletes (age $28.2 \pm 6.1$ years; height $179.2 \pm 5.9 \mathrm{~cm}$; weight 99.9 $\pm 16.5 \mathrm{~kg}$; body mass index $31.2 \pm 5.1$; body fat percentage 16.1 \pm 7.9 ) were examined before and after a 12-week supervised strength training period (SSTP). Study subjects have been exercised in powerlifting sports at competitive level from 4 to 15 years. Before the study, subjects completed a questionnaire concerning their personal and medical history and lifestyle parameters. All subjects were non-smokers and were free of acute or known chronic illnesses. In our study, only those voluntary subjects were included who agreed to avoid nutritional supplements. None of the participants were currently taking any medication. Subjects were asked not to take alcohol and not to change their usual diet during the SSTP. All subjects assessed their food intake three times during the study period for five consecutive days during the 12-week SSTP. All food items consumed were analyzed using the NutriData Estonian food composition database, version 8. One subject was excluded from data analysis due to the occurrence of injury and nonadherence to the full SSTP. Thus, the final study sample was 19 . Informed written consent was obtained from each athlete in accordance with the principles of the Declaration of Helsinki. The study protocol was approved by the Research Ethics Committee of the University of Tartu.

\section{Study protocol}

Before the SSTP, there was an 8-week off-season period, where systematic training sessions and competitions were not allowed. The pre-seasonal 12-week SSTP consisted of a combination of dynamic (where muscles contract concentrically during the lifting phase and eccentrically during the lengthening phase) and isometric (muscular force development without movement) resistance training. During the SSTP, one exercise session consisted of 5-8 working sets to one muscle group, and athletes exercised with an intensity of $60-90 \%$ assessed from 1 repetition maximum (1 RM). The first set was done with an intensity of $60 \%$ of $1 \mathrm{RM}$ and the last one $90 \%$ of $1 \mathrm{RM}$. Variation of repetitions was decreased according to the rise of intensity in one set (4 to 12). One exercise session lasted 90-120 min. The one-week training program (athletes exercised four days per week) consisted of the following exercises covering all major muscle groups: bench press, lateral pulldown, standing shoulder press, arm curl and extension, leg press, squat, leg curl, calf press, abdominal crunch, and deadlift. All the major muscle groups were exercised twice a week.

The structure of each exercise session was divided into three parts. The first part consisted of a 15-min warm-up, which included stretching and active exercises using body weight or very light weight. After the warm-up, the second part of the workout or the main training phase started, where the structure of the training session was on the ascending pyramid principle. ${ }^{28}$ This means that the first series was started with $60 \%$ of 1 RM with 12 repetitions, 
the second series was $70 \%$ of $1 \mathrm{RM}$ with 10 repetitions, and the third was $80 \%$ of $1 \mathrm{RM}$ with 8 repetitions. The fourth to sixth series were performed with loads ranging from $80 \%$ to $90 \%$ of 1 RM and $4-6$ repetitions were made. In one session, half of the muscle groups were influenced: 1) on Monday and Thursday exercises were performed on chest muscles, latissimus dorsi muscles, hamstring muscles, abdominal muscles, lower back muscles, and shoulder muscles; 2) on Tuesday and Friday exercises were performed on arm muscles (m. biceps brachii and m. triceps brachii), quadriceps muscles and calf muscles. The third part of the workout included a 15-min cooldown period, which included stretching and foam rolling for all muscles that experienced load in the training session. No aerobic exercise was involved in the training program and all the sessions were supervised by a skilled certified instructor. After the SSTP, all athletes in the study participated in the Estonian Powerlifting Championships. The training protocol used is characteristic for high-level powerlifters in the pre-seasonal phase.

\section{Measurements}

Anthropometric, hemodynamic, echocardiographic, and maximal muscular power measurements were taken one week before the SSTP while post-training period measurements were performed one week following the competitions. There were no training sessions on the day preceding the measurements (maximal muscular power, laboratory and arterial stiffness measurements). The hemodynamics (blood pressure, arterial stiffness) measurements were performed between 8 and 10 AM. Blood analyses were collected after hemodynamics measurements. For hemodynamic measurements and blood analysis, the subjects fasted and refrained from tea or coffee consumption overnight. Hemodynamics and echocardiographic examination were performed by a single experienced investigator.

\section{Anthropometric data}

Subjects' height and weight were determined by the Martin metal anthropometer $( \pm 0.1 \mathrm{~cm})$ and clinical scales $( \pm 0.05 \mathrm{~kg})$, and body mass index (BMI) was calculated $\left(\mathrm{kg} / \mathrm{m}^{2}\right)$. Body fat percentage was assessed by measuring skinfold thickness at six different anatomical sites. ${ }^{29}$

\section{Measurements of hemodynamics \\ Brachial blood pressure}

Brachial BP was measured in a sitting position from the non-dominant arm as a mean of three consecutive measurements at 5-min intervals using a validated oscillometric technique (OMRON M4-I; Omron Healthcare Europe BV, Hoofddorp, the Netherlands). The mean of the two closest BP readings was used in further analysis. ${ }^{30}$ Brachial pulse pressure was calculated as the difference between brachial systolic and diastolic BP.

\section{Central blood pressure and arterial stiffness}

Radial artery waveforms were recorded with a high-fidelity micromanometer (applanation tonometry) from the wrist of the dominant arm and pulse wave analysis was performed on the systolic portion (SphygmoCor, version 7.1, AtCor Medical, Sydney, Australia) of the pulse curve in accordance with established guidelines. ${ }^{31}$ The corresponding ascending aortic waveforms were then generated using a validated transfer function, ${ }^{31,32}$ from which central systolic and diastolic BP, central pulse pressure and augmentation index (AIx) were calculated. The AIx was defined as the difference between the second and the first systolic peaks of the central arterial waveform expressed as the percentage of central pulse pressure. ${ }^{31}$ Mean arterial pressure (MAP) was calculated from the integration of the radial artery waveform. The degree of pulse pressure amplification (PPA) was calculated as brachial pulse pressure divided by central pulse pressure.

\section{Carotid-femoral pulse wave velocity}

The carotid-femoral pulse wave velocity (cfPWV), a direct measure of aortic stiffness, was determined by sequential acquisition of pressure waveforms from the carotid and femoral arteries using the same tonometer (SphygmoCor, version 7.1, AtCor Medical). The timing of these waveforms was compared with that of the $\mathrm{R}$ wave on a simultaneously recorded ECG. The cfPWV was determined by a calculation of the difference between the carotid and the femoral path length divided by the difference in the $\mathrm{R}$ wave to waveform foot times. The difference between the carotid and the femoral path length was estimated from the distance of the sternal notch to the femoral pulse measured in a direct line. ${ }^{31}$ The within-observer coefficient of variation for cfPWV was $2.3 \%$.

The pulse wave analysis and cfPWV measurements were made in duplicate, and their mean values were used in subsequent analysis.

\section{Echocardiography}

An echocardiographic examination was performed using a commercially available device (Sonos7500, Philips Medical Systems, Inc., Highland Heights, OH, USA) with a $3.5-\mathrm{MHz}$ 
transducer and digital recording by an experienced cardiologist. The images were stored digitally, coded with a random number and read by a blinded observer. Left atrial dimension was measured using the two-dimensional echocardiography (2DE) anteroposterior linear method. For left ventricular mass calculations, the $2 \mathrm{DE}$ and the M-mode were used. Long-axis measurements were obtained at the level distal to the mitral valve leaflets. Left ventricular internal dimension and septal and posterior wall thickness were measured at the end of the diastole (subscript "d") according to the recommendations of the current guidelines. ${ }^{33}$ Left ventricular mass was calculated using the formula $0.8\left\{1.04\left[\left(\mathrm{LVID}_{\mathrm{d}}+\mathrm{PWT}_{\mathrm{d}}\right.\right.\right.$ $\left.\left.\left.+\mathrm{SWT}_{\mathrm{d}}\right)^{3}-\left(\mathrm{LVID}_{\mathrm{d}}\right)^{3}\right]\right\}+0.6 \mathrm{~g}\left(\mathrm{LVID}_{\mathrm{d}}-\right.$ left ventricular internal dimension, $\mathrm{PWT}_{\mathrm{d}}-$ posterior wall thickness, $\mathrm{SWT}_{\mathrm{d}}-$ septal wall thickness). ${ }^{33}$ For $2 \mathrm{DE}$ volume calculations, the modified Simpson's rule was used according to the current guidelines. ${ }^{33}$ For left ventricular diastolic function assessment, we measured medial E/e' ratio and mitral valve $\mathrm{E} / \mathrm{A}$ ratio in accordance with the recent guidelines. ${ }^{34}$ Left ventricular outflow tract velocity-time interval was measured with the pulsed-wave Doppler sample volume at the level of the left ventricular outflow tract in 3- or 5-chamber view. Tissue Doppler imaging (TDI) was performed in the apical fourchamber view. Peak systolic velocity of tricuspid annulus by TDI was measured as an indicator for right ventricular systolic function.

\section{Assessment of maximal muscular power}

Maximal muscular power was tested before and after the strength training period with bench press and jumping from a half squat (JHS) with a barbell. After the warmup, each athlete performed maximal effort in bench press and jumping from a half squat by the bar with his body weight following SSTP study protocol. The muscle power produced during squat jump was measured with a linear encoder attached to the barbell and fitted to the Musclelab system (Ergotest Innovation a.s., Porsgrunn, Norway). The method was introduced previously by Bosco et al. ${ }^{35}$

\section{Statistical analysis}

Statistical analysis was performed with SPSS for Windows software, version 22.0 (IBM Corporation, Armonk, NY, USA). All data were checked for normal distribution using the Kolmogorov-Smirnov test. Data are expressed as means (x) and standard deviations (SD). Carotid-femoral PWV was adjusted to MAP. The data were analyzed using the paired-samples $t$-test. The Pearson or Spearman correlations analysis was used to determine the relationships between the variables. For the observed power estimation, the general linear model, repeated measurements and power calculations were performed. Statistical significance was defined as two-sided $p<0.05$.

\section{Results}

Subjects' mean body weight, body fat percentage and BMI values after the 12-week strength training period did not differ significantly from baseline values $(99.9 \pm 16.5 \mathrm{vs}$ $100.2 \pm 17.1 \mathrm{~kg}, p=0.614 ; 16.1 \pm 7.9$ vs $15.8 \pm 7.7, p=0.906$; $31.2 \pm 5.1$ vs $31.3 \pm 5.2, p=0.603$, respectively). Table 1 provides descriptive information about the powerlifters' mean dietary intake during the study [total caloric intake, protein, fat, and carbohydrate intake in grams and total energy intake (\%) for proteins, fats and carbohydrates].

\section{Hemodynamic indices and blood pressure}

The hemodynamic indices at baseline and after the 12-week strength training period are presented in Table 2. Brachial and central systolic BP decreased significantly after the training period. There were no significant differences after the training period in cfPWV (both adjusted and unadjusted) and AIx (Table 2). After the SSTP, a slight decrease was detected in central pulse pressure and brachial pulse pressure, but the decrease remained nonsignificant. No significant changes were found in MAP, PPA (brachial pulse pressure/central pulse pressure), and heart rate after the SSTP.

\section{Echocardiography}

Echocardiographic parameters at baseline and after the 12-week strength training period are presented in Table 3. At baseline, left ventricular systolic function (ejection fraction $52-72 \%$ ) and mass index (49-115 $\left.\mathrm{g} / \mathrm{m}^{2}\right)$ were in normal range in all study subjects. However, increased left ventricular end-diastolic volume $\left(>64 \mathrm{~mL} / \mathrm{m}^{2}\right)$ was found in six subjects. Left atrial dimension and $\mathrm{E} / \mathrm{A}$ ratio

Table I Nutritional daily intake of the subjects

\begin{tabular}{|l|l|}
\hline Parameter & Mean I SD \\
\hline Total energy intake (kcal) & $4,200 \pm 845$ \\
Protein (g) & $190 \pm 60$ \\
Fat (g) & $168 \pm 56$ \\
Carbohydrate (g) & $495 \pm 147$ \\
Protein (\% energy) & $18.4 \pm 4.8$ \\
Fat (\% energy) & $35.5 \pm 4.8$ \\
Carbohydrate (\% energy) & $46.1 \pm 10.5$ \\
\hline
\end{tabular}

Notes: Data presented as means \pm SD. 
Table 2 Hemodynamic parameters of the subjects before and after the 12-week strength training period (SSTP)

\begin{tabular}{|l|l|l|l|}
\hline Parameter & $\begin{array}{l}\text { Before } \\
\text { SSTP }\end{array}$ & $\begin{array}{l}\text { After } \\
\text { SSTP }\end{array}$ & $\begin{array}{l}\boldsymbol{p} \text { - } \\
\text { value }\end{array}$ \\
\hline bSBP (mmHg) & $132.3 \pm 8.8$ & $124.3 \pm 8.7$ & 0.002 \\
bDBP (mmHg) & $70.1 \pm 5.9$ & $67.7 \pm 5.2$ & 0.059 \\
bPP (mmHg) & $61.2 \pm 8.4$ & $58.8 \pm 7.3$ & 0.259 \\
MAP & $88.4 \pm 7.4$ & $86.9 \pm 5.9$ & 0.359 \\
cSBP (mmHg) & $110.1 \pm 7.7$ & $104.5 \pm 8.7$ & 0.008 \\
cDBP (mmHg) & $71.1 \pm 6.3$ & $68.9 \pm 5,3$ & 0.078 \\
cPP (mmHg) & $39.0 \pm 3.7$ & $35.6 \pm 5.0$ & 0.317 \\
PPA & $1.6 \pm 0.1$ & $1.6 \pm 0.1$ & 0.650 \\
HR (b/min) & $62.6 \pm 8.6$ & $64.8 \pm 9.3$ & 0.093 \\
Alx (\%) & $3.3 \pm 9.2$ & $5.1 \pm 8.9$ & 0.233 \\
cfPWV (m/s) & $6.2 \pm 0.7$ & $6.5 \pm 1.1$ & 0.088 \\
Adjusted cfPWV (m/s) & $6.2 \pm 1.4$ & $6.4 \pm 1.9$ & 0.309 \\
\hline
\end{tabular}

Notes: Data presented as means \pm SD.

Abbreviations: bSBP, brachial systolic blood pressure; bDBP, brachial diastolic blood pressure; bPP, brachial pulse pressure; MAP, mean arterial pressure; cSBP, central systolic blood pressure; $c D B P$, central diastolic blood pressure; cPP, central pulse pressure; PPA, pulse pressure amplification; HR, heart rate; Alx, augmentation index; cfPWV, carotid-femoral pulse wave velocity.

remained unchanged after SSTP. However, E/e' ratio decreased significantly with $\operatorname{SSTP}(p<0.05)$. There was a statistically significant increase in tricuspid annular systolic velocity TDI $(p<0.01)$. An inverse correlation was found between the change in tricuspid annular systolic velocity TDI and the change in mean arterial pressure $(\mathrm{r}=-0.5 ; p<0.05)$.

\section{Maximal muscular power}

Descriptive data showed that mean values of bench press (BeP) and jumping from half squat (JHS) were significantly higher after the SSTP in comparison with baseline values (Figure 1), and these indices improved in all subjects. Observed power of effect size for BeP and JHS was between 0.41 and 0.46 for within-subjects effect and 0.96 and 1.0 for between-subjects effect, respectively.

\section{Discussion}

Resistance exercise has been shown to have a beneficial effect on muscular strength, bone mineral density, and body composition. ${ }^{36}$ Nevertheless, there is less evidence to demonstrate a protective effect of resistance exercise on cardiovascular health, particularly on BP and hemodynamic parameters, compared to aerobic exercise. ${ }^{37,38}$ The hemodynamic effect of resistance training differs significantly from aerobic training. In general, resistance training is divided into two subgroups having different effects on the cardiovascular system. Dynamic resistance training (force development associated with movement with intensity between $30 \%$ and $100 \%$ of $1 \mathrm{RM}$ ) has been shown to reduce BP and is recommended as a means for cardiovascular prevention in the hypertension guidelines. $^{24,39}$ Isometric resistance training (force development associated without movement with intensity between $10 \%$ and $40 \%$ of $1 \mathrm{RM}$ ) is not recommended for lowering $\mathrm{BP}$ because there are not enough studies available. ${ }^{24,39}$

Previous research indicates that isometric and isotonic exercise may have a differential effect on brachial and central blood pressure. $^{40-42}$ Indeed, central systolic blood pressure was increased only after hand-grip, but not after ergometer exercises. $^{40}$ It has been suggested that peripheral arterial dilatation caused by isotonic exercise might reduce the amplitude of reflected pressure waves. ${ }^{43}$ In contrast, isometric exercise could elevate arterial resistance, and thereby increase the amplitude of the radial reflected wave. ${ }^{43}$ In the present study, we found that strength training, which

Table 3 Echocardiographic parameters of the subjects before and after the 12-week strength training (SSTP)

\begin{tabular}{|c|c|c|c|}
\hline Parameter & Before SSTP & After SSTP & p-value \\
\hline Left ventricular ejection fraction (\%) & $63.5 \pm 4.5$ & $62.1 \pm 4.3$ & 0.056 \\
\hline Left ventricular end-diastolic volume/BSA $\left(\mathrm{mL} / \mathrm{m}^{2}\right)$ & $58.4 \pm 9.5$ & $57.7 \pm 10.5$ & 0.437 \\
\hline Cardiac output (L/min) & $5.6 \pm 0.9$ & $5.5 \pm 0.9$ & 0.703 \\
\hline Mitral valve E/A ratio & $1.6 \pm 0.4$ & $1.5 \pm 0.4$ & 0.319 \\
\hline IVS thickness (cm) & $1.0 \pm 0.2$ & $1.0 \pm 0.2$ & 0.447 \\
\hline Left atrial dimension $(\mathrm{cm})$ & $3.63 \pm 0.40$ & $3.58 \pm 0.35$ & 0.295 \\
\hline E/e' ratio & $7.8 \pm 1.3$ & $7.1 \pm 1.2$ & 0.026 \\
\hline LVMI $\left(g / \mathrm{m}^{2}\right)$ & $84.4 \pm 14.2$ & $84.5 \pm 17.3$ & 0.971 \\
\hline Relative wall thickness & $0.39 \pm 0.05$ & $0.40 \pm 0.07$ & 0.380 \\
\hline Tricuspid systolic annular velocity TDI, $\mathrm{cm} / \mathrm{s}$ & $13.0 \pm 2.3$ & $14.2 \pm 2.2$ & 0.006 \\
\hline
\end{tabular}

Notes: Data presented as means \pm SD.

Abbreviations: SSTP, supervised strength training program; BSA, body surface area; IVS, interventricular septal; LVMI, left ventricular mass index; TDI, tissue Doppler imaging. 


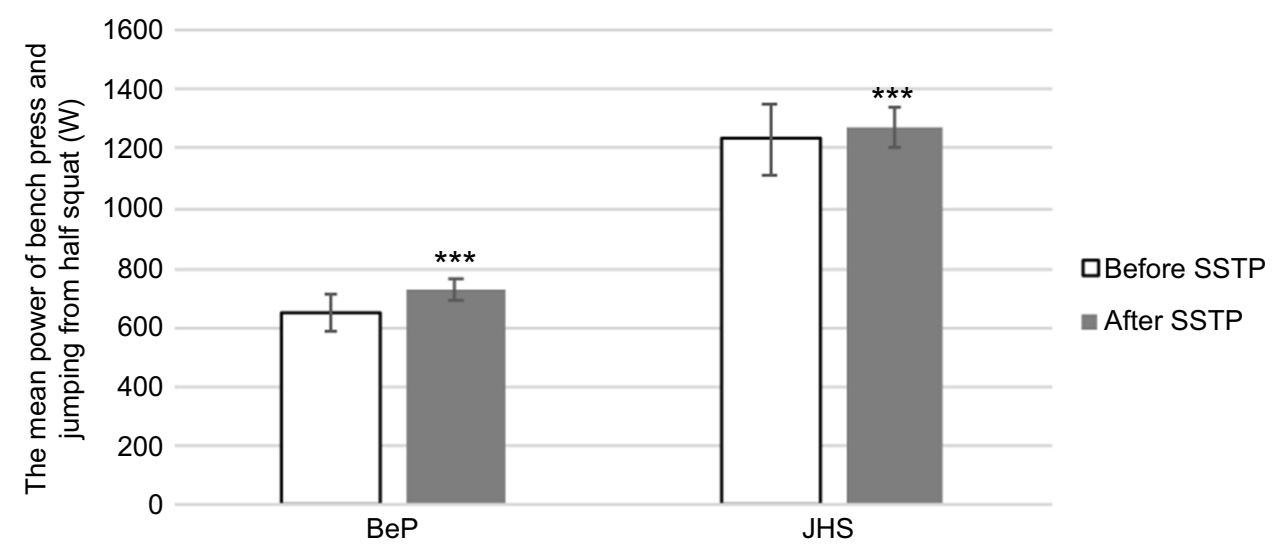

Figure I The mean power of bench press and jumping from half squat of the powerlifters before and after I2-week strength training period (SSTP). $* * * p<0.001$, in comparison to before SSTP.

Abbreviatons: BeP, bench press; JHS, jumping from half squat; SSTP, supervised strength training program.

combines dynamic and isometric resistance training with the intensity of $60-90 \%$ of $1 \mathrm{RM}$, resulted in a significant decrease in brachial and also in central systolic BP. However, Tanaka et al evaluated central blood pressure by Omron HEM-9000AI, which is based on the measurement of late systolic pressure of peripheral pressure wave. In our study, the SphygmoCor device was used, where the estimation of central blood pressure is based on the general transfer function. ${ }^{44}$ Moreover, the novelty of our study is that we evaluated central blood pressure and arterial stiffness in elite powerlifting athletes, whereas Tanaka et al studied clinically healthy subjects from the general population. ${ }^{40}$ In our study, subjects' mean brachial systolic and diastolic BP reduced by 8 and $2.4 \mathrm{mmHg}$, respectively. The results of our study support the evidence that strength training (combining dynamic and static exercise) has a significant BP-lowering effect in well-trained elite powerlifting athletes. In our study, the exposure to heavy training loads was supervised during the study period, and respective significant increases in maximal muscular power were revealed, which confirms that exposure to training loads was high in all subjects. Reduction of the peripheral and central blood pressure after resistance training could be explained by the change in the intensity and volume ratio of training. Despite prolonged intensive training, inactivity for several weeks can cause a loss of cardiovascular adaptation. Indeed, deconditioning is associated with a significant decrease in left ventricular wall thickness in highly trained athletes. ${ }^{45}$ Consistent with these findings, two months of inactivity resulted in a reduction of stroke volume, left ventricular end-diastolic diameter and cardiac output in highly trained subjects. ${ }^{46}$
Increased aortic stiffness is a well-known risk factor for cardiovascular disease. ${ }^{47}$ Controversy exists in the literature with regard to the effect of resistance exercise on aortic stiffness. Available mid- to long-term studies show a negative, ${ }^{17,18,25,48}$ neutral, ${ }^{26}$ or positive ${ }^{19,20}$ effect of resistance training on aortic stiffness. However, many of these studies were performed in relatively small samples in different age and sex groups and by a large variation in training intensity. Furthermore, most of the intervention studies were performed in untrained, recreational level or pre-hypertensive individuals. Most of the previously published study protocols are not comparable, especially where physical loads are very high. There are no data available for high-intensity strength training protocol, which is predominantly used by well-trained elite powerlifting athletes. In the present study, we did not find significant changes in cfPWV or AIx after the 12-week SSTP. It is possible that the changes in arterial stiffness were subtle because we studied well-trained powerlifters, who were conditioned for such strenuous training. In such a population, a 12-week training period might not be enough to evaluate the absolute effect of strength training on arterial stiffness. The BP-lowering effect of strength training is probably related to the reduction in peripheral vascular resistance ${ }^{27}$ and HR change, which moves the reflection point of the forward moving pulse wave more distally causing the BP to decrease.

Left ventricular hypertrophy is an independent cardiovascular risk factor in the general population ${ }^{49}$ and in hypertensive patients. ${ }^{50}$ In our study, there were no subjects with left ventricular hypertrophy, and there was no 
significant correlation between reduction in left ventricular mass and decrease in brachial BP. ${ }^{51}$ Our findings are consistent with previous research, where the 6-month resistance training did not have an effect on left ventricular remodeling. ${ }^{52}$ Furthermore, 12 weeks is a short period of time to observe structural changes in the heart. ${ }^{6}$ Left ventricular diastolic function can be used as an early marker of myocardial function change. In our study, SSTP improved left ventricular filling pressure, which indicates that change in central, not only peripheral BP, might improve left ventricular diastolic function. Moreover, to our knowledge, our study is the first one to show an increase in right ventricular systolic function and reduction in left ventricular filling pressure with resistance training. Assessment of diastolic function with echocardiography includes a complex of several variables; of these variables, E/e' ratio is the most frequently used method for left ventricular filling pressure assessment. ${ }^{33}$ Adler et al ${ }^{53}$ showed in an acute setting, resistance training increases $\mathrm{E} /$ A ratio and decreases deceleration time, which are both beneficial changes in terms of diastolic function. However, tissue Doppler was not used in this study. Two major factors influencing left ventricular filling pressure are left ventricular compliance and wall thickness. ${ }^{54}$ As our subjects' mean left ventricular wall thickness did not change significantly, it is possible that SSTP solely influenced their left ventricular compliance. While the left ventricle pumps into the systemic circulation, which has a significant reserve to decrease resistance and increase compliance with exercise, the right ventricle empties into the pulmonary circulation, which is characterized by low resistance and high compliance already at rest. Because there is a limited capacity to accommodate the blood flow in the pulmonary circulation with exercise, the right ventricle needs to maintain the necessary output. ${ }^{55}$ This explains the increase in the right ventricular systolic function parameter, ie, tricuspid annular systolic velocity TDI, in our study. Interestingly, we also found that an increase in right ventricular systolic function is associated with BP reduction. However, due to the design of our study, we cannot assess the causal relationship between these changes.

Our study has some limitations. There was no intention to involve a control group in this study due to several reasons. Firstly, it was not possible to sign elite level powerlifting athletes for 12 weeks without systematic strength training. Secondly, appropriate matched controls were not available for the present study group's characteristics because elite level powerlifters have a higher BMI as well as significantly higher muscle mass than other age- and weight-matched men. In addition, our study lasted for 12 weeks, which might be too short to assess the change in left ventricular mass. ${ }^{38}$

On the other hand, the novelty of our study is that it was performed in elite-level powerlifters and evaluates the effect of a real training load which is characteristic only for this target group. Thus, the results of our study provide more practical knowledge about the cardiovascular risk level for competitive powerlifters and other strength-oriented athletes.

Finally, further mechanistic studies should be undertaken to assess the relationship between strength training and right ventricular function. Thus, the findings of our study may have significant implications for future research.

\section{Conclusion}

The results of our study revealed that the 12-week pre-seasonal supervised strength training program (a combination of dynamic and isometric resistance training) significantly decreased brachial and central systolic blood pressure in male elite powerlifting athletes. The strength training program decreased left ventricular filling pressure and improved right ventricular systolic function. However, there was no effect of strength training on aortic stiffness.

\section{Acknowledgments}

The current study was supported by grants of Estonian Science Foundation (Nos. 7395 and 7480), Institutional Research Fundings (No. IUT 02-7 and PRG435) and target financing No. $0180105 \mathrm{~s} 08$ from the Ministry of Education and Research of Estonia. The authors wish to thank Helen Kaptein for her excellent technical assistance.

\section{Disclosure}

The authors report no conflicts of interest in this work.

\section{References}

1. Nichols WW, O'Rourke MF. McDonald's Blood Flow in Arteries: Theoretical, Experimental and Clinical Principles. 5th ed. London, United Kingdom: Arnold; 2005.

2. Corrado D, Basso C, Schiavon M, Pelliccia A, Thiene G. Pre-participation screening of young competitive athletes for prevention of sudden cardiac death. J Am Coll Cardiol. 2008;52(24):1981-1989. doi:10.1016/j.jacc.2008.06.053

3. Guo J, Zhang X, Wang L, Guo Y, Xie M. Prevalence of metabolic syndrome and its components among Chinese professional athletes of strength sports with different body weight categories. PLoS One. 2013;8e79758. doi:10.1371/journal.pone.0079758 
4. Berge $\mathrm{HM}$, Isern $\mathrm{CB}$, Berge $\mathrm{E}$. Blood pressure and hypertension in athletes: a systematic review. Br J Sports Med. 2015;49:716-723. doi:10.1136/bjsports-2014-093976

5. Volpe M, Battistoni A, Tocci G, et al. Cardiovascular risk assessment beyond systemic coronary risk estimation: a role for organ damage markers. $J$ Hypertens. 2012;30:1056-1064. doi:10.1097/HJ H.0b013e3283525715

6. Kampus P, Serg M, Kals J, et al. Differential effects of nebivolol and metoprolol on central aortic pressure and left ventricular wall thickness. Hypertension. 2011;57(6):1122-1128. doi:10.1161/HYPERT ENSIONAHA.110.155507

7. Roman MJ, Devereux RB, Kizer JR, et al. Central pressure more strongly relates to vascular disease and outcome than does brachial pressure: the strong heart study. Hypertension. 2007;50(1):197-203. doi:10.1161/HYPERTENSIONAHA.107.089078

8. Kals J, Kampus P, Kals M, et al. Inflammation and oxidative stress are associated differently with endothelial function and arterial stiffness in healthy subjects and in patients with atherosclerosis. Scand $J$ Clin Lab Invest. 2008;68(7):594-601. doi:10.1080/0036551 0801930626

9. Kampus P, Kals J, Unt E, et al. Association between arterial elasticity, C-reactive protein and maximal oxygen consumption in well-trained cadets during three days extreme physical load: a pilot study. Physiol Meas. 2008;29(4):429-437. doi:10.1088/0967-3334/ 29/4/001

10. Guerin AP, Blacher J, Pannier B, Marchais SJ, Safar ME, London GM. Impact of aortic stiffness attenuation on survival of patients in end-stage renal failure. Circulation. 2001;103:987-992.

11. Mora S, Cook N, Buring JE, Ridker PM, Lee IM. Physical activity and reduced risk of cardiovascular events: potential mediating mechanisms. Circulation. 2007;116(19):2110-2118. doi:10.1161/ CIRCULATIONAHA.107.729939

12. Higashi Y, Sasaki S, Kurisu S, et al. Regular aerobic exercise augments endothelium-dependent vascular relaxation in normotensive as well as hypertensive subjects: role of endothelium-derived nitric oxide. Circulation. 1999;100(11):1194-1202.

13. Ashor AW, Lara J, Siervo M, Celis-Morales C, Mathers JC, Li Y. Effects of exercise modalities on arterial stiffness and wave reflection: a systematic review and meta-analysis of randomized controlled trials. PLoS One. 2014;9(10):e110034. doi:10.1371/journal.pone.0110034

14. MacDougall JD, Tuxen D, Sale DG, Moroz JT, Sutton JR. Arterial blood pressure response to heavy resistance exercise. J Appl Physiol. 1995;58:785-790. doi:10.1152/jappl.1985.58.3.785

15. Lalande S, Baldi JC. Left ventricular mass in elite olympic weight lifters. Am J Cardiol. 2007;100:1177-1180. doi:10.1016/j. amjcard.2007.05.036

16. Pierce DR, Doma K, Leicht AS. Acute effects of exercise mode on arterial stiffness and wave reflection in healthy young adults: a systematic review and meta-analysis. Front Physiol. 2018;9:73. doi:10.3389/fphys.2018.00073

17. Miyachi M. Effects of resistance training on arterial stiffness: a metaanalysis. Br J Sports Med. 2013;47(6):393-396. doi:10.1136/ bjsports-2012-090488

18. Bertovic DA, Waddell TK, Gatzka CD, Cameron JD, Dart AM, Kingwell BA. Muscular strength training is associated with low arterial compliance and high pulse pressure. Hypertension. 1999;33 (6):1385-1391.

19. Morra EA, Zaniqueli D, Rodrigues SL, et al. Long-term intense resistance training in men is associated with preserved cardiac structure/function, decreased aortic stiffness, and lower central augmentation pressure. $J$ Hypertens. 2014;32(2):286-293. doi:10.1097/ HJH.0000000000000035

20. Okamoto T, Masuhara M, Ikuta K. Effects of low-intensity resistance training with slow lifting and lowering on vascular function. $J$ Hum Hypertens. 2008;22:509-511. doi:10.1038/jhh.2008.12
21. Jin YZ, Yan S, Yuan WX. Effect of isometric handgrip training on resting blood pressure in adults: a meta-analysis of randomized controlled trials. J Sports Med Phys Fitness. 2017;57(1-2):154-160. doi:10.23736/S0022-4707.16.05887-4

22. Inder JD, Carlson DJ, Dieberg G, McFarlane JR, Hess NC, Smart NA. Isometric exercise training for blood pressure management: a systematic review and meta-analysis to optimize benefit. Hypertens Res. 2016;39(2):88-94. doi:10.1038/hr.2015.111

23. Carlson DJ, Dieberg G, Hess NC, Millar PJ, Smart NA. Isometric exercise training for blood pressure management: a systematic review and meta-analysis. Mayo Clin Proc. 2014;89(3):327-334. doi:10.1016/j.mayocp.2013.10.030

24. Cornelissen VA, Smart NA. Exercise training for blood pressure: a systematic review and meta-analysis. $J$ Am Heart Assoc. 2013;2(1): e004473. doi:10.1161/JAHA.112.004473

25. Collier SR, Kanaley JA, Carhart R Jr, et al. Effect of 4 weeks of aerobic or resistance exercise training on arterial stiffness, blood flow and blood pressure in pre- and stage- 1 hypertensives. $J$ Hum Hypertens. 2008;22(10):678-686. doi:10.1038/jhh.2 008.36

26. Rakobowchuk M, McGowan CL, de Groot PC, et al. Effect of whole body resistance training on arterial compliance in young men. Exp Physiol. 2005;90(4):645-651. doi:10.1113/expphysiol.2004.029504

27. Umpierre D, Stein R. Hemodynamic and vascular effects of resistance training: implications for cardiovascular disease. Arq Bras Cardiol. 2007;89:256-262.

28. Fleck SJ, Kraemer WJ. Types of strength training. In: Designing Resistance Training Programs. 4th ed. Champaign, IL: Human Kinetics; 2014:15-62.

29. Tipton CM, Oppliger RA. The Iowa wrestling study: lessons for physicians. Iowa Med. 1984;74(9):381-385.

30. Mancia G, De Backer G, Dominiczak A, et al. ESH-ESC task force on the management of arterial hypertension. 2007 guidelines for the management of arterial hypertension: the task force for the management of arterial hypertension of the European Society of Hypertension (ESH) and of the European Society of Cardiology (ESC). $J$ Hypertens. 2007;25(6):1105-1187. doi:10.1097/ HJH.0b013e3281 fc975a

31. Mackenzie IS, Wilkinson IB, Cockcroft JR. Assessment of arterial stiffness in clinical practice. Qjm. 2002;95:67-74.

32. Pauca AL, O'Rourke MF, Kon ND. Prospective evaluation of a method for estimating ascending aortic pressure from the radial artery pressure waveform. Hypertension. 2001;38:932-937.

33. Lang RM, Badano LP, Mor-Avi V, et al. Recommendations for cardiac chamber quantification by echocardiography in adults: an update from the American society of echocardiography and the European association of cardiovascular imaging. Eur Heart $J$ Cardiovasc Imaging. 2015;16(3):233-270. doi:10.1093/ehjci/ jev014

34. Nagueh SF, Smiseth OA, Appleton CP, et al. Recommendations for the evaluation of left ventricular diastolic function by echocardiography: an update from the american society of echocardiography and the European association of cardiovascular imaging. $J$ Am Soc Echocardiogr. 2016;29(4):277-314. doi:10.1016/j.echo.2016.01.011

35. Bosco C, Belli A, Astrua M, et al. A dynamometer for evaluation of dynamic muscle work. Eur J Appl Physiol Occup Physiol. 1995;70 (5):379-386.

36. Gómez-Cabello A, Ara I, González-Agüero A, Casajús JA, VicenteRodríguez G. Effects of training on bone mass in older adults: a systematic review. Sports Med. 2012;42:301-325. doi:10.2165/ 11597670-000000000-00000

37. Cornelissen VA, Fagard RH. Effect of resistance training on resting blood pressure: a meta-analysis of randomized controlled trials. $J$ Hypertens. 2005;23(2):251-259. 
38. Yoon ES, Jung SJ, Cheun SK, Oh YS, Kim SH, Jae SY. Effects of acute resistance exercise on arterial stiffness in young men. Korean Circ J. 2010;40:16-22. doi:10.4070/kcj.2010.40.1.16

39. Mancia G, Fagard R, Narkiewicz K, et al. 2013 ESH/ESC guidelines for the management of arterial hypertension: the task force for the management of arterial hypertension of the European Society of Hypertension (ESH) and of the European Society of Cardiology (ESC). Eur Heart J. 2013;34(28):2159-2219. doi:10.1093/eurheartj/eht151

40. Tanaka S, Sugiura T, Yamashita S, Dohi Y, Kimura G, Ohte N. Differential response of central blood pressure to isometric and isotonic exercises. Sci Rep. 2014;4:5439. doi:10.1038/srep05439

41. Bentley DC, Nguyen CH, Thomas SG, Cindy HN. Resting blood pressure reductions following handgrip exercise training and the impact of age and sex: a systematic review and narrative synthesis. Syst Rev. 2018;7:229. doi:10.1186/s13643-018-0876-5

42. Vanhees L, Geladas N, Hansen D, et al. Importance of characteristics and modalities of physical activity and exercise in the management of cardiovascular health in individuals with cardiovascular risk factors: recommendations from the EACPR. Part II. Eur J Prev Cardiol. 2012;19(15):1005-1033. doi:10.1177/1741826711430926

43. Hashimoto J, Westerhof BE, Westerhof N, Imai Y, O’Rourke MF. Differential role of wave reflection magnitude and timing on left ventricular mass reduction during antihypertensivse treatment. J Hypertens. 2008;26:1017-1024. doi:10.1097/HJH.0b013e3282f62a9b

44. Butlin M, Qasem A. Large artery stiffness assessment using SphygmoCor technology. Pulse. 2016;4:180-192. doi:10.1159/ 000452448

45. Maron BJ, Pelliccia A, Spataro A, Granata M. Reduction in left ventricular wall thickness after deconditoning in highly trained athletes. Br Heart J. 1993;69:125-128. doi:10.1136/hrt.69.2.125

46. Martin WH, Coyle EF, Bloomfield SA, Ehsani AA. Effects of physical deconditioning after intense endurance training on left ventricular dimensions and stroke volume. J Am Coll Cardiol. 1986;7:982-989.
47. Payne RA, Wilkinson IB, Webb DJ. Arterial Stiffness and hypertension: emerging concepts. Hypertension. 2010;55:9-14. doi:10.1161/ HYPERTENSIONAHA.107.090464

48. Miyachi M, Kawano H, Sugawara J, et al. Unfavorable effects of resistance training on central arterial compliance: a randomized intervention study. Circulation. 2004;110(18):2858-2863. doi:10.1161/01. CIR.0000146380.08401.99

49. Levy D, Labib SB, Anderson KM, Christiansen JC, Kannel WB, Castelli WP. Determinants of sensitivity and specificity of electrocardiographic criteria for left ventricular hypertrophy. Circulation. 1990;81(3):815-820.

50. Koren MJ, Devereux RB, Casale PN, Savage DD, Laragh JH. Relation of left ventricular mass and geometry to morbidity and mortality in uncomplicated essential hypertension. Ann Intern Med. 1991;114:345-352.

51. Devereux RB, Dahlöf B, Gerdts E, et al. Regression of hypertensive left ventricular hypertrophy by losartan compared with atenolol: the Losartan Intervention for Endpoint Reduction in Hypertension (LIFE) trial. Circulation. 2004;110(11):1456-1462. doi:10.1161/01. CIR.0000141573.44737.5A

52. Spence AL, Naylor LH, Carter HH, et al. A prospective randomised longitudinal MRI study of left ventricular adaptation to endurance and resistance exercise training in humans. J Physiol. 2011;589 (22):5443-5452. doi:10.1113/jphysiol.2011.217125

53. Adler Y, Fisman EZ, Koren-Morag N, et al. Left ventricular diastolic function in trained male weight lifters at rest and during isometric exercise. Am J Cardiol. 2008;102(1):97-101. doi:10.1016/j. amjcard.2008.02.105

54. Mihl D, Dassen WR, Kuipers H. Cardiac remodelling: concentric vs eccentric hypertrophy in strength and endurance athletes. Neth Heart J. 2008;16(4):129-133.

55. La Gerche A, Heidbüchel H, Burns AT, et al. Disproportionate exercise load and remodeling of the athlete's right ventricle. Med Sci Sports Exerc. 2011;43(6):974-981. doi:10.1249/MSS.0b013e31820607a3
Research Reports in Clinical Cardiology

\section{Publish your work in this journal}

Research Reports in Clinical Cardiology is an international, peerreviewed, open access journal publishing original research, reports, editorials, reviews and commentaries on all areas of cardiology in the clinic and laboratory. The manuscript management system is completely online and includes a very quick and fair peer-review system. Visit http://www.dovepress.com/testimonials.php to read real quotes from published authors.

Submit your manuscript here: http://www.dovepress.com/research-reports-in-clinical-cardiology-journal 